\title{
Deformation-induced thermomagnetic effects in a twisted weak-link-bearing superconductor
}

\author{
Sergei A. Sergeenkov \\ Bogoliubov Laboratory of Theoretical Physics, Joint Institute for Nuclear Research, \\ 141980 Dubna, Russia
}

(October 31, 2018)

\begin{abstract}
Based upon the recently introduced thermophase and piezophase mesoscopic quantum effects in Josephson junctions, several novel phenomena in a twisted superconductor (containing a small annular $S I S$-type contact) under influence of thermal gradient and applied magnetic field are predicted. Namely, we consider a torsional analog of Josephson piezomagnetism (and related magnetomechanical effect) as well as a possible generation of a heat flux induced magnetic moment in a weaklycoupled superconductor under a torsional deformation (analog of Zavaritskii effect) along with the concomitant phenomena of piezothermopower and piezothermal conductivity. The conditions under which the predicted effects can be experimentally measured in conventional superconductors and nanostructured materials with implanted Josephson contacts are discussed.
\end{abstract}

In 1972 Zavaritskii [1] observed for the first time a very interesting phenomenon (the so-called deformation-induced thermomagnetic effect): appearance of a heat flux $Q$ induced magnetic field $\Delta H=H_{q}(\alpha) Q$ in rod-like tin samples (both in the normal and superconducting state) under a torsional deformation $\mathcal{M}$ (related to a torsional angle $\alpha(\mathcal{M})=\mathcal{M} / C_{0}$, where $C_{0}$ is a respective elastic modulus of the material). Quite a tangible value of $\Delta H$ was registered under the maximum load of $\mathcal{M}=0.2 \mathrm{~N} / \mathrm{m}$ (which corresponds to $\alpha=0.01 \mathrm{rad} / \mathrm{cm}$ ). This phenomenon was attributed to generation of circular (non-potential) currents in a deformed sample (which in turn lead to observable magnetic moments, see Ref.2 for discussion) and further investigated by Lebedev [3] on the basis of the kinetic theory.

At the same time, in response to fast growing interest to important applications of Josephson and proximity effects in novel mesoscopic quantum devices (such as, e.g., quantum computers), a substantial progress has been made recently in measuring of (and manipulating with) extremely small magnetic fields, thermal gradients and mechanical deformations [4.5].

Based upon the recently introduced thermophase [6.7] and piezophase [8.9] effects (suggesting, respectively, a direct influence of a thermal gradient and an applied stress on phase difference through a Josephson junction), in this Letter we discuss an analog of the above-mentioned Zavaritskii effect in a twisted superconductor containing a single SIStype contact and its possible realization in conventional superconductors. Besides, we also consider the concomitant phenomena of Josephson piezomagnetism and magnetomechanical effect as well as the change of transport properties of $S I S$-type junction under torsional deformation (piezothermopower and piezothermal conductivity).

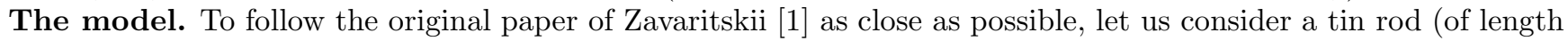
$L$ and radius $R$ ) with an anular $S n-S n O-S n$ contact [10] incorporated into the middle of the rod (due to a high pliability of tin, it should be quite easy to achieve), with a thin insulating $S n O$ layer (of thickness $l$ ). Assuming a usual cylindrical geometry (with $z$-axis taken along the rod's length and $A=\pi R^{2}$ being the junction area), we can present the total Josephson energy on the contact as follows (for the sake of simplicity, in this paper we shall concentrate on zero-temperature effects only and will ignore the role of Coulomb interaction effects assuming that the grain's charging energy $E_{c} \ll E_{J}$, where $E_{c}=e^{2} / 2 C_{J}$, with $C_{J}$ being the capacitance of the junction):

$$
E_{J}=\int_{0}^{\tau} \frac{d t}{\tau} \int_{-R}^{R} \frac{d x}{A} \int_{-\sqrt{R^{2}-x^{2}}}^{\sqrt{R^{2}-x^{2}}} d y \int_{0}^{L} \frac{d z}{L}[\mathcal{H}(\vec{x}, t)]
$$

where the local Josephson energy is given by

$$
\mathcal{H}(\vec{x}, t)=J[1-\cos \phi(\vec{x}, t)]
$$

with the resulting phase difference

$$
\phi(\vec{x}, t)=\phi_{0}+\frac{2 \pi d B x}{\Phi_{0}}+\alpha(\mathcal{M}) z+\frac{2 \pi S_{0} \nabla T \vec{x}}{\Phi_{0}} t
$$

accounting for the change of the initial phase difference $\phi_{0}$ under the influence of an applied magnetic field $\vec{B}=$ $(0, B, 0)$, thermal gradient $\nabla T=\left(\nabla_{x} T, 0, \nabla_{z} T\right)$ and applied torsional deformation $\mathcal{M}$ (through the corresponding 
torsional angle $\alpha(\mathcal{M})$ ) taken along $z$-axis. Here $\Phi_{0}=h / 2 e$ is the quantum of flux with $h$ Planck's constant and $e$ the electronic charge, $d=2 \lambda_{L}+l$ is the junction size with $\lambda_{L}$ being the London penetration length, $\tau$ is a characteristic Josephson time [9], $J$ is the Josephson coupling energy, and $S_{0}$ is the field-free thermoelectric power (Seebeck coefficient) on the junction.

The origin of the third term in Eq.(3) is quite obvious. Indeed, under the influence of an homogeneous torsional deformation $\mathcal{M}$, the superconducting phase difference will change with $z$ as follows: $d \phi / d z=(d \phi / d \theta)(d \theta / d z)=N \alpha(\mathcal{M})$ where 11] $\alpha(\mathcal{M}) \equiv d \theta / d z=\mathcal{M} / C_{0}$ is the corresponding torsional angle variable and $N \equiv d \phi / d \theta$ is a geometrical factor (in most cases [8] $N \simeq 1$ ). As a result, the superconducting phase difference will acquire an additional contribution $\delta \phi(z)=\alpha(\mathcal{M}) z$. (Notice that practically the same result can be obtained by using the arguments from Ref.9 and invoking an analogy with a conventional linear torsional piezoelectric effect which predicts [12] $P(\mathcal{M})=a \mathcal{M}$ for an induced electric polarization.)

To neglect the influence of the self-field effects and ensure a uniformity of the applied deformation $\mathcal{M}$ (and the related torsional angle $\theta(L) \equiv \alpha(\mathcal{M}) L$ ), we have to assume that $\lambda_{J}>R$ and $L \gg R$ where $\lambda_{J}=\sqrt{\Phi_{0} / 2 \pi d \mu_{0} j_{c}}$ is the Josephson penetration depth with $j_{c}$ being Josephson critical current density. As we shall see below, these conditions can be reasonably well met experimentally.

Torsional piezomagnetic effect. Before turning to the main subject of this paper, let us briefly discuss the two preliminary issues: (i) deformation induced behavior of the Josephson current and (ii) torsional analog of Josephson piezomagnetism (which takes place in a twisted SIS-type contact and manifests itself through appearance of deformation-induced susceptibility) in the absence of thermal gradient through the junction $(\nabla T=0)$. Recalling the definition of the Josephson current density $j_{s}(\vec{x})=j_{c} \sin \phi(\vec{x}, 0)$, in this particular case Eq.(3) brings about

$$
I_{s}(B, \theta)=2 I_{c}\left[\frac{J_{1}\left(B / B_{0}\right)}{B / B_{0}}\right]\left(\frac{\sin \theta}{\theta}\right)
$$

for the maximum (with $\phi_{0}=\pi / 2$ ) Josephson current in a twisted cylindrical contact (under a torsional deformation $\mathcal{M}$ producing angle $\theta=\mathcal{M} L / C_{0}$ ) with $I_{c}=j_{c} A=2 e J / \hbar$. Here $B_{0}=\Phi_{0} / 2 \pi d R$ is a characteristic Josephson field of anular contact, and $J_{1}(x)$ is the Bessel function. Notice that as a function of torsional angle $\theta$, the induced current $I_{s}(B, \theta)$ follows a quasi-periodic Fraunhofer-like pattern and reduces to the well-known [13] result for the magnetic field dependence of an anular Josephson contact upon removal of mechanical load (in deformation-free case when $\theta \rightarrow 0)$.

Moving on to the second issue, we find that in addition to the above-discussed angle-dependent Josephson current, in a twisted contact will appear an induced magnetic moment (torsional piezomagnetic effect)

$$
M_{s}(B, \theta) \equiv-\frac{1}{V}\left[\frac{\partial E_{J}}{\partial B}\right]_{\nabla T=0}
$$

where $V=A L$ is a sample's volume.

To capture the very essence of this effect, in what follows we assume for simplicity that an unloaded sample does not possess any spontaneous magnetization at zero magnetic field (that is $M_{s}(0,0)=0$ ) and that its Meissner response to a small applied field $B$ is purely diamagnetic (that is $M_{s}(B, 0) \simeq-B$ ). According to Eqs.(1)-(5), this condition implies $\phi_{0}=2 \pi m$ for the initial phase difference with $m=0, \pm 1, \pm 2, \ldots$ As a result, we obtain for the change of magnetization under torsional deformation

$$
M_{s}(B, \theta)=-M_{0} f_{1}\left(B / B_{0}\right) g_{0}(\theta)
$$

Here $M_{0}=2 J / V B_{0}, f_{1}(x)=-\frac{d}{d x}\left[f_{0}(x)\right]$ with $f_{0}(x)=J_{1}(x) / x$, and $g_{0}(x)=\sin x / x$.

For low-field (Meissner) region, we can linearize the above equation and define the deformation induced angledependent susceptibility $\chi(\theta)$. Indeed, for $B \ll B_{0}$, Eq.(6) gives $M_{s}(B, \theta) \simeq \chi(\theta) B$ where $\chi(\theta)=-\chi_{0} g_{0}(\theta)$ with $\chi_{0}=J / 4 V B_{0}^{2}$. As it follows from the above equations, the superconducting (Meissner) phase of piezomagnetization $M_{s}(B, \theta)$ (and the corresponding susceptibility $\chi(\theta)$ ) gradually dwindles with increasing the angle $\theta$, shifting towards paramagnetic phase (and reaching it eventually at $\theta \simeq \pi$ ).

Magnetomechanical effect. Let us consider the converse (to piezomagnetism) magnetomechanical effect, that is field induced change of torsional angle $\theta_{s}(B, \mathcal{M})$ (and corresponding compliance $C_{s}^{-1}(B)$, see below). In view of Eqs.(1)-(3), we obtain

$$
\theta_{s}(B, \mathcal{M}) \equiv\left[\frac{\partial E_{J}}{\partial \mathcal{M}}\right]_{\nabla T=0}=\theta_{0} f_{0}\left(B / B_{0}\right) g_{1}\left(\mathcal{M} / \mathcal{M}_{0}\right)
$$


where $\theta_{0}=J / \mathcal{M}_{0}$ with $\mathcal{M}_{0}=C_{0} / L$ and $g_{1}(x)=-\frac{d}{d x}\left[g_{0}(x)\right]$.

Notice that in the absence of applied magnetic field (when $B=0$ ) the above equation establishes the so-called "torque-angle" relationship (torsional analog of "stress-strain" law [8]) for a twisted weak-link-bearing superconductor $\theta_{s}(0, \mathcal{M})=\theta_{0} g_{1}\left(\mathcal{M} / \mathcal{M}_{0}\right)$. For small enough torsional deformations (when $\mathcal{M} \ll \mathcal{M}_{0}$ which is usually the case in realistic experiments), the above model relationship reduces to a more familiar Hooke's law, $\theta_{s}(0, \mathcal{M})=\mathcal{M L} / C_{s}(0)$ with $C_{s}(0)=\left(3 C_{0} / J L\right) C_{0}$ being the appropriate (zero-field) elastic modulus (inverse torsional compliance) whose magnetic field dependence is governed by the following equation

$$
\frac{1}{C_{s}(B)} \equiv \frac{1}{L}\left[\frac{\partial \theta_{s}(B, \mathcal{M})}{\partial \mathcal{M}}\right]_{\mathcal{M}=0}=\frac{1}{C_{s}(0)}\left[\frac{2 J_{1}\left(B / B_{0}\right)}{B / B_{0}}\right]
$$

It would be rather interesting to try to observe the above-discussed piezomagnetic and magnetomechanical effects (including torsional analog of paramagnetic Meissner effect [9]) in a twisted weak-link-containing superconductor.

Deformation induced thermomagnetic effects. Let us turn now to the main subject of this paper and consider the influence of a thermal gradient $\nabla T$ on the above discussed piezomagnetic and magnetomechanical effects. Hereafter we restrict our consideration to the case of small values of the applied thermal gradient $\nabla T$, leading to linear thermoelectric effects (for discussion of possible nonlinear Seebeck effects in Josephson junctions and granular superconductors see Ref.7). For thermal gradient applied normally and parallel to the torsional deformation (see Eq.(3)), we obtain two contributions (transverse and longitudinal) for the deformation induced thermomagnetization, emerging in the vicinity of the Josephson contact

$$
\Delta M(\theta, B, \nabla T) \equiv-\frac{1}{V}\left[\frac{\partial E_{J}}{\partial B}\right]_{\nabla T \neq 0}=M_{\perp}^{q}(\theta, B) \nabla_{x} T+M_{\|}^{q}(\theta, B) \nabla_{z} T
$$

where

$$
M_{\perp}^{q}(\theta, B)=M_{0 \perp}^{q} f_{2}\left(B / B_{0}\right) g_{0}(\theta)
$$

and

$$
M_{\|}^{q}(\theta, B)=M_{0 \|}^{q} f_{1}\left(B / B_{0}\right) g_{1}(\theta)
$$

Here $M_{0 \|}^{q}=(L / R) M_{0 \perp}^{q}$ with $M_{0 \perp}^{q}=2 e J S_{0} \tau R / B_{0} V \hbar$, and $f_{2}(x)=\frac{d}{d x}\left[f_{1}(x)\right]$.

Notice that within the geometry adopted in the present paper, the true analog of Zavaritskii effect is given by a zero field limit of the transverse (paramagnetic) contribution, $M_{\perp}^{q}(\theta, 0)=(1 / 8) M_{0 \perp}^{q}(\sin \theta / \theta)$. Its evolution with torsional angle follows the corresponding behavior of the induced susceptibility $\chi(\theta)$ (Cf. Eq.(6)) and, thus, it also undergoes a "diamagnetic-paramagnetic" transition upon reaching a critical angle $\theta_{c} \simeq \pi$. On the other hand, the field-dependent longitudinal component $M_{\|}^{q}(\theta, B)$ changes with $\theta$ linearly for $\theta \ll 1$. It describes the appearance of deformation induced thermomagnetic component normal to the applied magnetic field $\vec{B}=(0, B, 0)$. It disappears when $B \rightarrow 0$ and for non-zero fields it closely follows the behavior of the (initially diamagnetic) torsional piezomagnetization $M_{s}(B, \theta)$ considered in the previous section (Cf. Eq.(6)).

Likewise, for thermal gradient applied normally and parallel to the torsional deformation (see Eq.(3)), we obtain two contributions for the heat flux induced magnetomechanical effect

$$
\Delta \theta(\mathcal{M}, B, \nabla T) \equiv\left[\frac{\partial E_{J}}{\partial \mathcal{M}}\right]_{\nabla T \neq 0}=\theta_{\perp}^{q}(\mathcal{M}, B) \nabla_{x} T+\theta_{\|}^{q}(\mathcal{M}, B) \nabla_{z} T
$$

where

$$
\theta_{\perp}^{q}(\mathcal{M}, B)=\theta_{0 \perp}^{q} f_{1}\left(B / B_{0}\right) g_{1}\left(\mathcal{M} / \mathcal{M}_{0}\right)
$$

and

$$
\theta_{\|}^{q}(\mathcal{M}, B)=\theta_{0 \|}^{q} f_{0}\left(B / B_{0}\right) g_{2}\left(\mathcal{M} / \mathcal{M}_{0}\right)
$$

Here $\theta_{0 \|}^{q}=(L / R) \theta_{0 \perp}^{q}$ with $\theta_{0 \perp}^{q}=2 e S_{0} J R \tau / \hbar \mathcal{M}_{0}$ and $g_{2}(x)=-\frac{d}{d x}\left[g_{1}(x)\right]$.

Once again, the true (deformation-free) heat flux induced magnetomechanical effect is given by the zero-deformation limit of the longitudinal component $\theta_{\|}^{q}(0, B)$ while a small angle expansion of the transverse component describes a thermal analog of Hooke's law $\theta_{\perp}^{q}(\mathcal{M}, B) \simeq \mathcal{M} / C_{\perp}^{q}(B)$ with $C_{\perp}^{q}(B)$ being the appropriate compliance coefficient. 
Piezothermopower. Let us briefly discuss now one more interesting phenomenon of piezothermopower which can occur in a twisted superconducting rod with a SIS-type junction. According to Eqs.(1)-(3), the transverse and longitudinal contributions to (magnetic field-dependent) change of junction's Seebeck coefficients under torsional deformation read:

$$
\Delta S_{\perp}(\theta, B) \equiv-\frac{1}{e R}\left[\frac{\partial E_{J}}{\partial \nabla_{x} T}\right]=S_{J} f_{1}\left(B / B_{0}\right) g_{0}(\theta)
$$

and

$$
\Delta S_{\|}(\theta, B) \equiv-\frac{1}{e L}\left[\frac{\partial E_{J}}{\partial \nabla_{z} T}\right]=-S_{J} f_{0}\left(B / B_{0}\right) g_{1}(\theta)
$$

with $S_{J}=(2 \tau J / \hbar) S_{0}$.

Like in the previous paragraph, the deformation-free transversal component $\Delta S_{\perp}(0, B)$ describes the evolution of conventional thermopower in unloaded $(\theta=0)$ sample with applied magnetic field, while the true piezothermopower is given by a zero-field limit of the longitudinal component $\Delta S_{\|}(\theta, 0)$. Besides, the above analysis allows us to introduce a deformation induced AC Josephson effect (as a generalization of a more familiar thermal AC effect [14]) in a twisted $S I S$-contact bearing superconductor. Indeed, as soon as the thermal current exceeds the critical current, which will happen when $\Delta T$ becomes larger than $\Delta T_{c}(\theta, B)=I_{c} R_{n} / \Delta S_{\|}(\theta, B)$ (where $R_{n}$ is the normal state resistance), a new type of Josephson generation with frequency $\omega(\theta, B)=2 e \Delta S_{\|}(\theta, B) \Delta T / \hbar$ should occur in the junction area (see below for more discussion).

Piezothermal conductivity. Finally, let us briefly consider the influence of mechanical deformation on magnetothermal conductivity of the twisted weak-link-bearing superconducting rod. To this end, we recall that the local heat flux density $\vec{q}(\vec{x}, t)$ is related to the local Josephson energy density $\mathcal{H}(\vec{x}, t)$ via the energy conservation law

$$
\nabla \vec{q}(\vec{x}, t)+\dot{\mathcal{H}}(\vec{x}, t)=0
$$

where $\dot{\mathcal{H}}=\partial \mathcal{H} / \partial t$.

The above equation allows us to introduce an effective thermal flux

$$
\vec{Q}(t) \equiv \frac{1}{V} \int d^{3} x \vec{q}(\vec{x}, t)=\frac{1}{V} \int d^{3} x \dot{\mathcal{H}}(\vec{x}, t) \vec{x}
$$

which in turn is related to the thermal conductivity tensor $\kappa_{\alpha \beta}$ as follows $(\{\alpha, \beta\}=x, y, z)$

$$
<Q_{\alpha}>\equiv \frac{1}{\tau} \int_{0}^{\tau} d t Q_{\alpha}(t)=-\kappa_{\alpha \beta} \nabla_{\beta} T
$$

Straightforward calculations based on Eqs.(1)-(3) yield the following explicit expressions for non-zero components of the thermal conductivity tensor

$$
\begin{aligned}
& \kappa_{x x}(\theta, B)=\kappa_{0}\left(\frac{R}{L}\right) J_{1}^{\prime}\left(B / B_{0}\right) g_{x x}(\theta) \\
& \kappa_{x z}(\theta, B)=\kappa_{z x}(\theta, B)=\kappa_{0} J_{1}\left(B / B_{0}\right) g_{x z}(\theta) \\
& \kappa_{z z}(\theta, B)=\kappa_{0}\left(\frac{L}{R}\right) J_{0}\left(B / B_{0}\right) g_{z z}(\theta)
\end{aligned}
$$

where $\kappa_{0}=8 e S_{0} J R L / h V, J_{1}^{\prime}(x)=J_{0}(x)-J_{1}(x) / x$ with $J_{n}(x)$ being the corresponding Bessel functions, $g_{x x}(x)=$ $(1-\cos x) / x, g_{x z}(x)=\frac{d}{d x}\left[g_{x x}(x)\right]$, and $g_{z z}(x)=-\frac{d^{2}}{d x^{2}}\left[g_{x x}(x)\right]$.

For small torsional deformations (with $\theta \ll 1), g_{x x}(\theta) \simeq \theta / 2, g_{x z}(\theta) \simeq\left(1-\theta^{2} / 4\right) / 2$ and $g_{z z}(\theta) \simeq \theta / 4$. So, $\kappa_{x z}(0, B)$ describes a conventional (deformation-free) magnetothermal conductivity in unloaded junction [15], while true (magnetic field-free) piezothermal conductivity is given by $\kappa_{x x}(\theta, 0)$ and $\kappa_{z z}(\theta, 0)$ components (both increasing linearly with $\theta$ for small angles). In a sense, introduced here piezothermal conductivity is a converse effect with respect to the original Zavaritskii effect [1.2] and it seems very interesting to try to realize it experimentally using tin rods (with and without weak links).

Discussion. To estimate the magnitudes of the predicted effects, we make use of the fact that for thermoelectric processes the characteristic time $\tau$ is related to a thermal AC frequency $\omega_{T}=2 e S_{0} \Delta T / \hbar$ (assuming $\nabla_{x} T \simeq \Delta T / R$ 
and $\nabla_{z} T \simeq \Delta T / L$ ) as follows: $\tau=2 \pi / \omega_{T}$. As a result, the deformation induced thermomagnetic field (Josephson analog of Zavaritskii effect) reads:

$$
\Delta H_{\perp} \equiv 4 \pi M_{\perp}^{q}(\theta, 0) \nabla_{x} T \simeq H_{J}\left(\frac{R}{L}\right)\left(\frac{\sin \theta}{\theta}\right)
$$

where $\mu_{0} H_{J}=\Phi_{0} / 2 \pi \lambda_{J}^{2}$ is a critical Josephson field.

Furthermore, combining the experimental parameters for $S n-S n O-S n$ anular Josephson junction considered by Matisoo 10] (namely, $R_{n}=10^{-9} \Omega, j_{c}(0)=10^{4} \mathrm{~A} / \mathrm{m}^{2}, \lambda_{L}(0)=40 \mathrm{~nm}$, and $\lambda_{J}(0)=5 \mathrm{~mm}$ ) with the typical parameters from Zavaritskii experiments on tin rods [1.2] (namely, $2 R=5 \mathrm{~mm}, L=8 \mathrm{~cm}$, and $\theta_{\max }=0.1 \mathrm{rad}$ ) we obtain $\Delta B_{\perp}=\mu_{0} \Delta H_{\perp} \simeq 10^{-12} \mathrm{~T}$ which is equivalent to quite a tangible value of the deformation induced thermomagnetic flux through the junction $\Delta \Phi_{\perp}=\pi R^{2} \Delta B_{\perp} \simeq 10^{-2} \Phi_{0}$. The same set of parameters yields $B_{0}=$ $\Phi_{0} / 2 \pi d R \simeq 10^{-6} T$ and $B_{J}=\Phi_{0} / 2 \pi \lambda_{J}^{2} \simeq 10^{-10} T$ for estimates of characteristic and critical Josephson fields, respectively. Finally, using some typical [14] experimental data describing physics of conventional Josephson contacts (with $S_{0} \simeq 10^{-8} V / K$ and $\Delta T \simeq 10^{-4} K$ ), we can estimate the orders of magnitude of the piezothermal conductivity and deformation induced AC Josephson generation (related to piezothermopower). The result is as follows, $\kappa_{z z}(\theta, 0) \simeq$ $\left(2 e S_{0} J L^{2} / h V\right) \theta \simeq(2 / \pi) S_{0} j_{c} \theta \simeq 10^{-2} W / m K$ and $\omega(\theta, 0)=2 e \Delta S_{\|}(\theta, 0) \Delta T / \hbar \simeq\left(\omega_{J} \tau / 3\right) \theta \omega_{T} \simeq 10^{11} H z$, where $\omega_{J}=$ $2 J / \hbar \simeq 10^{12} \mathrm{~Hz}$ and $\omega_{T}=2 e S_{0} \Delta T / \hbar \simeq 1 k H z$ are characteristic (Josephson and thermal, respectively) frequencies. The above estimates suggest quite an optimistic possibility to observe the predicted here effects experimentally through a comparative study of conventional superconductors with and without $S I S$-type junctions.

At the same time, to check the validity of some other interesting predictions (like Fraunhofer patterns given by a set of $g_{n}(\theta)$ functions, see the text), much larger torsional deformations (reaching critical angles of the order of $\theta_{c} \simeq \pi$ ) are needed. Hopefully, this will become possible in the very nearest future, with further advancement of experimental techniques and new technologies for manufacturing of nanostructured superconducting materials with implanted atomic scale Josephson junctions and other weak links 顿].

In conclusion, a few novel mesoscopic quantum phenomena which are expected to occur in a weak-link-bearing superconductor under influence of torsional deformation, thermal gradient and applied magnetic field were presented. The observability of the predicted effects, using conventional superconductors as well as novel materials with wellcontrolled mesoscopic Josephson junctions was discussed.

The idea of this work was conceived and partially realized during my stay at the Universidade Federal de São Carlos (Brazil) where it was funded by the Brazilian Agency FAPESP (Projeto 2000/04187-8). I thank Wilson Ortiz and Fernando Araujo-Moreira for hospitality and stimulating discussions on the subject.

[1] N.V. Zavaritskii, JETP Lett. 16, 67 (1972).

[2] N.V. Zavaritskii, Sov. Phys. JETP 40, 601 (1975).

[3] V.V. Lebedev, Sov. Phys. JETP 40, 577 (1975).

[4] For recent reviews on the subject, see, e.g., Mesoscopic and Strongly Correlated Electron Systems, Eds. V.F. Gantmakher and M.V. Feigel'man, Phys.Usp. 41, N2 (1998); Mesoscopic and Strongly Correlated Electron Systems-II, Eds. M.V. Feigel'man, V.V. Ryazanov and V.B. Timofeev, Phys. Usp. (Suppl.) 44, N10 (2001).

[5] S. Sergeenkov, in Studies of High Temperature Superconductors, vol. 39, Eds. A. Narlikar and F. Araujo-Moreira, Nova Science, New York, 2001, pp. 117-131.

[6] G.D. Guttman, B. Nathanson, E. Ben-Jacob et al., Phys. Rev. B 55, 12691 (1997).

[7] S. Sergeenkov, JETP Lett. 67, 680 (1998).

[8] S. Sergeenkov, J. Phys.: Cond. Mat. 10, L265 (1998).

[9] S. Sergeenkov, JETP Lett. 70, 36 (1999).

[10] J. Matisoo, J. Appl. Phys. 40, 1813 (1969).

[11] L.D.Landau and E.M.Lifshitz, Theory of Elasticity, Pergamon Press, New York,1960.

[12] L.D. Landau and E.M. Lifshitz, Electrodynamics of Continuous Media, Pergamon Press, New York, 1984.

[13] A.Barone and G.Paterno, Physics and Applications of the Josephson Effect, A Wiley-Interscience Publisher, New York, 1982.

[14] G.I. Panaitov, V.V. Ryazanov, A.V. Ustinov et al., Phys. Lett. A 100, 301 (1984).

[15] S. Sergeenkov and M. Ausloos, Phys. Rev. B 48, 4188 (1993). 